\title{
La Evaluación del Desempeño Docente desde la perspectiva del docente y de los administradores del proceso
}

\author{
Claudia Lina Esmeralda Villa Arias ${ }^{1}$ \\ Sonia Espinoza Morales ${ }^{2}$ \\ Briseida Sandoval González ${ }^{3}$
}

\begin{abstract}
1https://orcid.org/0000-0001-9706-2643. cvillaa@ittepic.edu.mx. Tecnológico Nacional de México/Instituto Tecnológico de Tepic

${ }^{2}$ https://orcid.org/0000-0002-1260-667. sespinoza@ittepic.edu.mx. Tecnológico Nacional de México/Instituto Tecnológico de Tepic
\end{abstract}

${ }^{3}$ https://orcid.org/0000-0002-4132-7400. bsandoval@ittepic.edu.mx. Tecnológico Nacional de México/Instituto Tecnológico de Tepic

\section{DOI: https://doi.org/10.46589/rdiasf.vi35.402}

Recibido 13 de Marzo 2021.

Aceptado 26 de junio 2021

Publicado 30 de junio de 2021

\section{Resumen}

Este proyecto se desprende de la línea de investigación educativa del Instituto Tecnológico de Tepic, denominada Gestión Académica, la cual está alineada a los objetivos e indicadores institucionales, y creada para fortalecer las competencias del perfil de los docentes del Tecnológico Nacional de México. El instrumento analizado es utilizado para evaluar el desempeño de los docentes adscritos a uno de los 126 Institutos Tecnológicos Federales o a uno de los 122 Institutos Tecnológicos Descentralizados del país, todos adscritos al Tecnológico Nacional de México (TecNM). Con este proyecto de investigación se espera contribuir a la mejora continua del proceso educativo, por ello es importante que se incorpore la opinión del docente como sujeto evaluado a este proceso, para incorporar su perspectiva y generar propuestas de mejora a futuro ya sea en los ISSN: 2007-8870 
instrumentos, procesos del sistema de evaluación, mecanismos o el proceso en general. Se presenta parte de la segunda etapa de un proyecto realizado y concluido en el Instituto Tecnológico de Tepic, plantel educativo considerado para el estudio de este proyecto; con esto, no sólo se cuenta con la percepción de los estudiantes, también se considera para futuros proyectos y mejoras la opinión del docente, actor principal de la evaluación.

\section{Introducción}

Para que un proceso pueda ser mejorado debe ser evaluado, encaminado y dirigido a la mejora continua, cuando se evalúa la forma de trabajo de las personas, lejos de verse como algo inquietante o molesto, debe recibirse como una oportunidad de recibir objetivamente las observaciones y comentarios que puedan surgir de ellas. Durante años, las instituciones de Educación Superior han implementado sistemas de evaluación al desempeño del personal docente, y el Tecnológico Nacional de México (TecNM) no es la excepción, para evaluar a sus docentes hace uso de dos tipos de evaluaciones: la Evaluación Departamental, elaborada a través de su área académica; y la Evaluación del Desempeño Docente, que cada fin de semestre es realizada por los estudiantes quienes evalúan el desempeño de sus docentes, siendo esta última el eje central de este proyecto de investigación.

Este trabajo intenta captar la apreciación que se tiene en el Tecnológico Nacional de México campus Tepic, de la Evaluación del Desempeño Docente desde la perspectiva del docente y de los administradores del proceso, aunque para efecto de este documento sólo se incluyen los resultados de los cuestionarios aplicados a los docentes. Este proyecto tiene como finalidad mejorar y retroalimentar el sistema de evaluación, sus mecanismos, instrumentos y procesos; previo a esta investigación se llevó a cabo el análisis desde la perspectiva del estudiante, información que en conjunto será utilizada en una tercera fase para llevar a cabo un estudio comparativo.

El sentido formativo del docente de alta calidad no podría concebirse sin que exista una mejora continua, en relación a esto se puede decir que el sistema de evaluación del desempeño docente permite obtener información importante para mejorar el servicio que se ofrece a los estudiantes, estimular a los profesores para que realicen los cambios pertinentes y reconocerles su trabajo cuando los resultados sean favorables. A lo largo de la investigación se realizan varios 
cuestionamientos, mismos que dan sentido y dirección al proyecto de investigación; asimismo, se intenta identificar la opinión que tienen los docentes respecto a la modalidad de aplicación, diseño del instrumento, validez de sus resultados, impacto de los resultados obtenidos y retroalimentación de la misma, entre otros aspectos.

Planteamiento del problema

Las organizaciones más comprometidas y competitivas en la actualidad reconocen que la capacitación además de ser un agente motivador, es parte de un proceso de mejora continua que permite que las personas evolucionen; como consecuencia si los docentes reciben la capacitación pertinente podrán fortalecer su actuar docente y mejorar la formación profesional de los estudiantes, sin olvidar el beneficio y prestigio que se forma alrededor de las instituciones. La evaluación del desempeño es uno de los medios que puede proporcionar información suficiente para la mejora continua; y para que los procesos que se encuentran al interior de las instituciones educativas aseguraren la educación de calidad. Es necesario innovar o en su caso adoptar nuevas tendencias que se orienten a la calidad en la educación en todos los niveles nacional e internacional. Para esto, es necesario observar de manera sistémica los mecanismos establecidos para evaluar el desempeño del docente, quien es un actor estratégico en el proceso de enseñanza-aprendizaje.

Es importante destacar que para la realización de la investigación, se contó con el apoyo de estudiantes del área de Ciencias Económico Administrativas, con ello, se fortalece la formación integral de los estudiantes y se les permite aplicar y desarrollar los conocimientos que tienen en el área de investigación.

Justificación.

El modelo educativo para el siglo XXI del SNIT hoy TecNM (2012), establece como uno de sus objetivos estratégicos, ofrecer servicios educativos de calidad. Una de las características de la gestión educativa para el alto desempeño implantada en el Tecnológico Nacional México, es que “promueve la capacitación y el desarrollo de competencias, así como la evaluación del desempeño del personal de la institución” (DGEST, p.72).

1SSN: $2007-8870$

Universidad de Sonora
Los contenidos de este articulo ertin bajo una licendia de Creative Commons Atribución No Comercial-Sin Obra Derivada 4 , Ointernaciona! 
El actual Plan Nacional de Desarrollo 2019-2024 aborda el tema de educación en el eje general de Bienestar donde se plasmaron dos objetivos principales, un indicador y sus siete estrategias que direccionan todas sus acciones del Sistema Educativo Nacional hacia la inclusión, igualdad, calidad y cobertura en todos los niveles educativos, en el objetivo 2.2. se menciona que se debe garantizar a todas las personas, su derecho a la educación laica, gratuita, incluyente, pertinente y de calidad en todos los tipos, niveles y modalidades; asimismo, se plantea el interés por fortalecer la calidad de la educación a través de la mejora de los procesos y el desempeño de los docentes, considerando la formación, capacitación y actualización parte esencial para fortalecer la profesionalización docente, con lo cual también, se podrá mejorar la selección, promoción y reconocimiento de los docentes (Gobierno de la Republica, 2019).

En la página 37 del Programa de Trabajo Anual del Tecnológico Nacional de México 2019, se menciona en el objetivo 1, que se debe fortalecer la calidad de los servicios educativos, mientras que en la Estrategia 2, se indica que se debe reconocer el desempeño docente del profesorado promoviendo la participación en actividades docencia, investigación, vinculación y gestión académica; con ello promueve que el profesor cumpla con todas las actividades de docencia consideradas en la evaluación del desempeño docente (PTA TecNM, n.d.).

La evaluación de la efectividad de la docencia es un aspecto que se considera fundamental en la mayoría de las Instituciones de Educación Superior; contribuir a la calidad de la educación con que se llevan a cabo las funciones docente es indispensable, por lo tanto la evaluación del desempeño docente se ha convertido en una de las herramientas más importante en la toma de decisiones, con estos resultados es posible detectar las áreas de oportunidad o mejora, así como identificar las fortalezas para reconocer de alguna manera la eficacia de un proceso especifico. Este sistema de evaluación del desempeño docente debe proporcionar información oportuna y confiable, que ofrezca realmente la retroalimentación adecuada a los docentes y a los administradores del proceso y en nuestro caso debe estar al mismo tiempo alineado a los objetivos institucionales y acorde a las competencias del perfil docente del Tecnológico Nacional de México. Como parte de los atributos que el docente debe tener para contribuir a tener una educación de calidad no se puede 
prescindir de un sistema eficaz y eficiente de la evaluación de su desempeño, ya que se promueve la mejora continúa haciendo de forma efectiva la retroalimentación de los resultados.

En este estudio se analizará la opinión del docente y de los administradores del proceso de evaluación, la utilidad de los resultados, el grado de aceptación del instrumento de evaluación, con el objetivo de generar propuestas de mejora a futuro a la evaluación del desempeño. Con base a lo anteriormente expuesto se considera que es pertinente el estudio del tema; además, se cuenta con los recursos humanos, técnicos y documentales necesarios para su realización, así como el apoyo institucional para llevar a cabo la aplicación de los instrumentos necesarios.

Objetivo general del proyecto:

Analizar el proceso de evaluación al desempeño docente desde la perspectiva de los docentes y de los administradores del proceso del Instituto Tecnológico de Tepic.

\section{Objetivos específicos:}

1. Describir la opinión del docente en relación al instrumento de evaluación docente, considerando el diseño del cuestionario, modalidad y momento de la evaluación.

2. Identificar el impacto de los resultados de la evaluación del desempeño de los docentes.

3. Identificar la utilidad de los resultados de la evaluación del desempeño docente para los administradores del proceso.

Antecedentes

La evaluación del desempeño en las IES (Instituciones de Educación Superior) en México surge desde hace más de tres décadas en algunas universidades privadas, en el caso de las universidades públicas, la evaluación se implementa de forma generalizada a partir de las políticas promovidas por la Secretaría de Educación Pública (SEP) y la Asociación Nacional de Universidades e Instituciones de Educación superior (ANUIES) en la década de 1990. Cada una de las instituciones de educación superior en México tiene un sin fin de actividades diversas que están vinculadas a las continuas evaluaciones, los principales que están involucrados en la educación son los profesores 
y estudiantes, en esa interacción se generan acciones que son sujetas de evaluación. En los años noventa la evaluación formaba parte del Plan de Educación, esto ha evolucionado y se han generado acciones estratégicas con la finalidad de mejorar la calidad de la educación. A partir de esto se implementaron políticas sustentadas en la evaluación dando lugar a los programas, procesos y acciones, estas políticas siguen vigentes y con el tiempo se han ido ajustando de acuerdo a cada Institución de Educación Superior.

Se observa que, en el contexto de la política educativa nacional y la calidad de los servicios ofrecidos por las Instituciones Educación Superior, un elemento clave es el papel desempeñado por los docentes, de ahí el interés por evaluar su actividad. Atender a la valoración de las actividades de los profesores se justifica ante su finalidad que es la de mejorar la enseñanza, a través de la retroalimentación proporcionada a los docentes y lograr la actualización permanente a través de la capacitación continua, derivada de los resultados obtenidos en las mismas evaluaciones.

Las acciones de evaluación al desempeño del personal académico en México se caracterizan por programas de compensación salarial como el Sistema Nacional de Investigadores (surgido en 1984), el programa de estímulos al desempeño docente (en 1990) y el Programa de Mejoramiento del Profesorado (desde 1997), y otros programas especiales promovidos en algunas IES.

A partir del análisis de los procesos de evaluación de la docencia de seis universidades públicas: la Nacional Autónoma de México (UNAM), la Autónoma Metropolitana (UAM), la Autónoma de Querétaro (UAQ), la Autónoma del Estado de Hidalgo (UAEH), la Autónoma de Baja California (UABC) y la Autónoma del Estado de Morelos (UAEM), Rueda, Elizalde y Torquemada (2003) identificaron que en la mayoría de las IES la evaluación de los docentes solo es considerada para el acceso a los programas de compensación salarial; en algunas universidades, sobre todo de nueva creación, se utiliza la evaluación docente como parte de los procedimientos de ingreso del personal; se privilegia la investigación antes que la docencia; las IES no cuentan con dependencias o centros de operación definidos en los organigramas oficiales para el tema de la evaluación docente; en las universidades con amplia matrícula se delega en las escuelas y facultades el diseño y aplicación de la evaluación docente; predomina la evaluación docente desde 
la opinión de los estudiantes; y por último, en algunas IES complementan la evaluación con cuestionarios aplicados al jefe inmediato y con la autoevaluación del profesor

De esta manera se resume lo más sobresaliente de la evaluación del desempeño docente en las universidades públicas de la Ciudad de México, así como algunos aspectos positivos que pudieran ser adoptados. La presencia de este tipo de evaluación es universal y está asociada a los programas de compensación económica y acreditación, lo que confirma la influencia de las políticas públicas de evaluación. Predomina la evaluación del desempeño docente mediante la opinión de los estudiantes, obtenida a través de cuestionarios; ésta supone una alta especialización de conocimiento técnico de quienes asumen esta responsabilidad, por lo que las universidades deben facilitar la participación de especialistas y el desarrollo de programas de formación profesional, sin embargo son pocos los esfuerzos de evaluación vinculados con acciones institucionales de apoyo a los académicos para mejorar su labor como docentes. Aun así, en muchas otras universidades de educación superior no se aplican acciones institucionales para mejorar el desempeño docente pues se ven limitados en recursos y disposición del propio docente (Rueda, Elizalde y Torquemada, 2003).

Evaluar la docencia siempre ha sido parte de la política vigente para todos los institutos que integraban la Dirección General de Educación Superior Tecnológica (DGEST), ahora Tecnológico Nacional de México (TecNM), en cada institución se elaboraban y aplicaban diversos instrumentos para obtener información y con los cuales se podía acreditar la eficiencia de la docencia. La diversidad de variables consideradas para evaluar a los profesores era muy amplia, en algunos casos se tomaban en cuenta variables de presagio, es decir características propias del profesor como su experiencia docente, el grado de estudios, motivación e incluso rasgos de personalidad. Esta variedad en la integración de instrumentos daba de alguna manera resultados útiles a cada institución, pero impedía tener una visión global de la eficiencia de la docencia a nivel Sistema. Con el propósito de tener información estandarizada para reconocer la eficiencia de la docencia en el Sistema de Educación Tecnológica, el Consejo del Sistema Nacional de Educación Tecnológica se hizo cargo de la evaluación docente desde 1992 hasta 2005. Durante estos años, en la construcción del instrumento de evaluación se tomaron en cuenta la planeación del curso, dominio 
de contenidos de la materia, vinculación teoría práctica, disponibilidad del acervo bibliográfico, uso de material didáctico, motivación, evaluación del aprendizaje, relación profesor-alumno y responsabilidad del profesor.

El Modelo Educativo para el Siglo XXI, incorpora la evaluación como una actividad básica, con las siguientes características: es un proceso continuo de realimentación para estudiantes, profesores y autoridades; atiende criterios e indicadores de las disciplinas y del desempeño profesional; asegura la equidad y el logro de los propósitos formativos; valora los procesos y los productos del aprendizaje. La evaluación y sus resultados constituyen áreas de oportunidad para desarrollar programas de intervención tanto para docentes como para estudiantes. Actualmente el TecNM identifica y reconoce en el docente un conjunto de competencias básicas que, al declararse, definen en forma explícita el desempeño del docente. Las competencias vigentes del perfil docente declaradas en el Cuestionario de Evaluación docente con enfoque en competencias son: disciplina, planificación del curso, diseño de ambientes de aprendizaje, estrategias, métodos y técnicas de aprendizaje, motivación, evaluación del aprendizaje, comunicación, gestión del curso, tecnologías de la información y de la comunicación, y se añade un apartado relacionado con la Satisfacción general del curso (DGEST, 2013).

Para una mejor apreciación de los conceptos de desempeño y evaluación, se presentan varias definiciones, la primera es la que se obtiene de la Real Academia Española que define al "desempeño" como la acción de desempeñar o desempeñarse; y, "desempeñar" es cumplir las obligaciones inherentes a una profesión, cargo u oficio. Por otra parte, para García (2001) el desempeño representa; "aquellas acciones o comportamientos observados en los empleados que son relevantes para los objetivos de la organización, y que pueden ser medidos en términos de las competencias de cada individuo y su nivel de contribución a la empresa" (p.3). Por lo tanto, el concepto de desempeño se puede definir como la utilidad o la productividad que una persona en el ejercicio de las actividades que tiene que ejecutar puede aportar a una organización. Ahora, la evaluación es un concepto que puede definirse de diferentes formas dependiendo de las necesidades o propósitos de la persona u organismo que requiere de la medición, la evaluación exige un proceso 
metodológico que permita valorar desde la perspectiva cualitativa y cuantitativa los resultados obtenidos.

De igual manera Fernández (2006), considera que la evaluación debe ser un procesos sistematizado, fundamentado técnicamente y diseñado para obtener información confiable, objetiva y valida, que permita una toma de decisión pertinente. La evaluación es una función que, formal o informalmente, siempre se aplica en todos los niveles jerárquicos de una organización con el objeto de lograr una mejora de la actividad de cada empleado inserto en ella, mediante la observación de su comportamiento en su puesto de trabajo; el procedimiento que mide el desempeño de los empleados en su puesto de trabajo se denomina comúnmente evaluación del desempeño y lo podemos entender como la función que observa los comportamientos del empleado en el ejercicio de las funciones de su puesto con el propósito de lograr mejoras en su desempeño.

Al respecto, Certo (1994) describe la evaluación del desempeño como “el proceso de revisar la actividad productiva anterior con el objeto de evaluar que tanto contribuyeron los individuos a que se alcanzaran los objetivos del sistema administrativo" (p.280). El proceso de la evaluación del desempeño es conocido por diferentes nombres, como calificación de méritos, evaluación del rendimiento, evaluación de la eficiencia personal, etc. Según Werther - Davis (2000), "la Evaluación del Desempeño constituye el proceso por el cual se estima el rendimiento global del empleado. Constituye una función esencial que de una u otra manera suele efectuarse en toda organización moderna" (p.231). Mientras que para Chiavenato (2009), la evaluación del desempeño es "una valoración sistemática de la actuación de cada persona en función de las actividades que desempeña, las metas y los resultados que debe alcanzar, las competencias que ofrece y su potencial de desarrollo" (p. 245).

Por lo anterior, la evaluación del desempeño debe ser considerada como una herramienta fundamental para cualquier organización o institución y, principalmente para los directivos que quieran conducir a esta por el camino de la calidad, ya que la evaluación del desempeño es el procedimiento para evaluar el recurso humano y generalmente se elabora a partir de programas formales de evaluación basados en una razonable cantidad de información respecto de los empleados y de su desempeño en el puesto, esto con el fin de reforzar las debilidades de los 
trabajadores a través de capacitaciones, o de igual forma sirve a las organizaciones para promocionar a los empleados, reubicarlos o premiarlos. Todo lo anterior, con el propósito de lograr mayor productividad en todo tipo de institución, sean estas industriales, comerciales o de servicios, o públicas o privadas.

El desempeño del docente se entiende como el ejercicio y cumplimiento de sus responsabilidades; el cual se encuentra determinado por elementos ligados al propio docente, a los estudiantes, al sistema educativo y al entorno. De igual manera, el desempeño se ejerce en diferentes campos; el contexto socio-cultural, el entorno institucional, el ambiente de aula y sobre el propio docente. El desempeño se evalúa para mejorar la calidad educativa y cualificar la profesión docente. Algunos estudiosos de la materia argumentan que la definición de desempeño debe ser completada con la descripción de lo que se espera de los empleados, además de una continua orientación hacia el desempeño efectivo. Para Juárez (2018) el desempeño es un proceso que debe planearse, dirigirse, controlarse y, en su momento, rediseñarse para que responda a las exigencias de los objetivos, las metas y los resultados esperados a corto, mediano y largo plazo. Entonces, conviene tener claro que el proceso de evaluación del desempeño es un medio o herramienta y no un fin en sí mismo.

La evaluación del desempeño es una valoración sistemática de la actuación de cada persona en función de las actividades que desempeña, las metas y los resultados que debe alcanzar, las competencias que ofrece y su potencial de desarrollo, es un proceso que sirve para juzgar y estimar, la excelencia y las competencias de una persona, pero, sobre todo, la aportación que hace al negocio de la organización (Chiavenato, 2008). En este sentido, Gómez-Mejía (2008) refiere que la evaluación del desempeño consiste en identificar, medir y administrar el desempeño humano en las organizaciones. La identificación se apoya en el análisis de los puestos y pretende determinar cuáles áreas del trabajo se deben estudiar cuando se mide el desempeño. La medición es el elemento central del sistema de evaluación y pretende determinar cómo ha sido el desempeño en comparación con ciertos parámetros objetivos. La administración es el punto central de todo sistema de evaluación y debe ser mucho más que una actividad que se orienta al pasado; por el contrario, para desarrollar todo el potencial humano de la organización, se debe orientar hacia el 
futuro, para lo cual se debe tener antecedentes del comportamiento y desempeño de su capital humano, con ello, será posible modificar conductas y formas de trabajo a través de programas de formación y actualización; sin olvidar que, los resultados, la retroalimentación y el análisis de todo ello puede ser una parte de la propia autocorrección, esmero, atención, entrenamiento personal y automotivación de las personas evaluadas. Al respecto, Robalino (2005) define la evaluación del desempeño como; "el proceso de movilización de sus capacidades profesionales, su disposición personal y su responsabilidad social para: "articular relaciones significativas entre los componentes que impactan la formación de los alumnos; participar en la gestión educativa; fortalecer una cultura institucional democrática, e intervenir en el diseño, implementación y evaluación de políticas educativas locales y nacionales, para promover en los estudiantes aprendizajes y desarrollo de competencias y habilidades para la vida" (p. 11).

Si analizamos los principales usos que las organizaciones dan a los resultados de la evaluación del desempeño podremos encontrar que las utilizan para asignar compensaciones, detectar necesidades de capacitación y formación, identificar candidatos a posibles promociones o incluso a probables despidos, evaluación de procesos y actualización de inventarios de capacidades y/o competencias humanas de la organización (Juárez, 2018). Existen pocos estudios sobre evaluación del desempeño en las universidades; sin embargo, los existentes, nos han permitido identificar que estos procesos no son cien por ciento exitosos, probablemente por la desconfianza que se tiene en ellos. Por ello, es relevante trabajar en la sensibilización de las partes involucradas para que vean a la evaluación como un medio para mejorar el desempeño, como una oportunidad para identificar las fortalezas de los docentes y a partir de ahí crear políticas públicas que permitan la generalización y estandarización de las mismas (Tejedor, 2012).

En México se reporta el uso de los cuestionarios de apreciación estudiantil como una estrategia utilizada por el $98 \%$ de las instituciones encuestadas, de las cuales el $86 \%$ evalúa mediante este medio al total de la población de docentes y $12 \%$ los usa para evaluar a una muestra de profesores (Arámburo y Luna, 2013); sin embargo, existe muy poca investigación al respecto de la retroalimentación del proceso de evaluación. La evaluación del desempeño docente tiene propósito formativo y se sustenta en la necesidad de conocer la práctica de los profesores en los 
escenarios educativos — aulas, talleres, laboratorios, espacios de práctica- para decidir qué aspectos deben reforzarse, mejorarse o modificarse en las dimensiones disciplinarias, pedagógicas, tecnológicas, comunicativas o éticas del ejercicio de los profesores (Gómez y Valdés, 2019).

La Evaluación Educativa representa una cultura, práctica y política institucional bastante tardía en el avance y fortalecimiento de la educación formal en México, mientras que la Evaluación del Desempeño Docente en la educación obligatoria, constituye una de las áreas con menor grado de desarrollo en el Sistema Educativo Nacional, con respecto a la amplia experiencia generada en el contexto internacional (Guzmán, 2018). En el Instituto Tecnológico de Tepic, se tiene como referente una investigación relacionada con el Desempeño docente, realizada por García Torres, Espinoza Morales y Sandoval González (2017), su objetivo fue determinar la relación que existe entre los resultados de la evaluación al desempeño docente realizada por los estudiantes y la experiencia docente en el aula, tomando como base los años de servicio en la actividad docente (antigüedad), así como la formación pedagógica y disciplinar del docente determinada por los cursos de actualización acumulados por cada docente en los últimos cinco años. Los resultados mostraron que no existe evidencia estadística suficiente para comprobar que la calificación obtenida en la evaluación docente tiene relación con el número de cursos tomados por los docentes en el período comprendido entre el año 2010 y el año 2014. Por otro lado, tampoco se pudo afirmar que la calificación tiene relación con la antigüedad (en años) que tiene el docente impartiendo clases en el Instituto Tecnológico de Tepic. Es de considerarse que este estudio es un precedente en el análisis de la evaluación al desempeño docente en el Instituto Tecnológico de Tepic, que hace reflexionar acerca del tratamiento de los resultados de la evaluación y la toma de decisiones en cuanto a la capacitación pertinente para cada docente, según su área de formación, edad y antigüedad. Sin embargo, resulta necesario analizar los mecanismos de evaluación, para lograr que el proceso de evaluación docente sea eficaz y eficiente.

\section{Metodología}

El cuestionario de Evaluación del Desempeño Docente del Tecnológico Nacional de México campus Tepic analizado, consta de 10 dimensiones correspondiendo las primeras 9 a las 
competencias docentes (dominio de la asignatura, planificación del curso, ambientes de aprendizaje, estrategias, métodos y técnicas, motivación, evaluación, comunicación, gestión del curso y tecnologías de la información y la comunicación) y una dimensión adicional para la evaluación del grado de satisfacción general que el estudiante manifiesta sobre el docente. En la Tabla 1, se muestra la distribución del número de enunciados por dimensión.

\section{Tabla 1}

Estructura de instrumento de la Evaluación al Desempeño Docente

\begin{tabular}{lcc}
\hline Dimensión & Enunciados & Cantidad \\
\hline Dominio de la asignatura & $01-05$ & 5 \\
Planificación del curso & $06-08$ & 3 \\
Ambientes de aprendizaje & $09-13$ & 5 \\
Estrategias, métodos y técnicas & $14-20$ & 7 \\
Motivación & $21-27$ & 7 \\
Evaluación & $28-35$ & 8 \\
Comunicación & $36-38$ & 3 \\
Gestión del curso & $39-42$ & 4 \\
Tecnologías de la información y comunicación & $43-45$ & 3 \\
Satisfacción general & $46-48$ & 3 \\
\hline
\end{tabular}

Para establecer el tamaño de la muestra de 167 docentes, se consideró una población de 299 docentes distribuidos en nueve áreas académicas, en donde se recurrió a la técnica de investigación de campo virtual para recoger su opinión. En la Tabla 2 se muestra la distribución de los docentes por departamento académico, en la Tabla 3, se puede apreciar los datos de la población y la muestra dada. 
Año 14 / Núm. 35 / Enero-junio 2021

Revista de Investigación Académica sin Frontera

ISSN 2007-8870

\section{Tabla 2}

Distribución de docentes

\begin{tabular}{|c|c|c|c|c|}
\hline Departamentos & Docentes & Porcentajes & $\begin{array}{l}\text { Docentes que } \\
\text { contestaron la } \\
\text { encuesta }\end{array}$ & $\begin{array}{l}\text { Cantidad proporcional de } \\
\text { docentes que deben contestar la } \\
\text { encuesta }\end{array}$ \\
\hline Ingeniería Industrial & 23 & $7,69 \%$ & 14 & 13 \\
\hline Arquitectura & 42 & $14,05 \%$ & 24 & 23 \\
\hline Posgrado & 16 & $5,35 \%$ & 11 & 9 \\
\hline $\begin{array}{l}\text { Ciencias Básicas } \\
\text { Ciencias Económico }\end{array}$ & 30 & $10,03 \%$ & 17 & 17 \\
\hline Administrativas & 65 & $21,74 \%$ & 46 & 36 \\
\hline $\begin{array}{l}\text { Ingeniería Civil } \\
\text { Ingeniería En Sistemas }\end{array}$ & 36 & $12,04 \%$ & 20 & 20 \\
\hline $\begin{array}{l}\text { Computacionales } \\
\text { Ingeniería Química Y }\end{array}$ & 40 & $13,38 \%$ & 22 & 22 \\
\hline Bioquímica & 25 & $8,36 \%$ & 17 & 14 \\
\hline \multirow[t]{2}{*}{ Ingeniería Eléctrica } & 22 & $7,36 \%$ & 12 & 12 \\
\hline & 299 & $100 \%$ & 183 & 167 \\
\hline
\end{tabular}

\section{Tabla 3}

Datos de la muestra y población

\begin{tabular}{lll}
\hline Símbolo & Descripción & Datos \\
\hline $\mathrm{N}=$ & población & 299 \\
$\sigma=$ & nivel de confianza & $95 \%$ \\
$\mathrm{p}=$ & probabilidad a favor & $50 \%$ \\
$\mathrm{q}=$ & probabilidad en contra & $50 \%$ \\
$\mathrm{n}=$ & muestra & 167 \\
$\mathrm{e}=$ & error & $5 \%$ \\
\hline
\end{tabular}

$$
\mathrm{n}=\frac{\sigma^{2} N p q}{\mathrm{e}^{2}(N-1)+\sigma^{2} p q}=
$$

$1.96^{2}(299)(.50)(.50)$

$.05^{2}(299-1) 1.96^{2}(.50)(.50)$

$=167$ 
Para el análisis de los resultados se obtuvieron 183 personas encuestadas y nueve administradores del proceso, así como al coordinador general del proceso, respecto a la participación específica, conocimiento y aceptación que se tiene del instrumento de evaluación del desempeño docente y su aplicación. Los docentes o administradores del proceso están asignados y se encuentran organizados de la siguiente manera: Arquitectura, Ciencias Básicas, Ciencias Económico - Administrativas, Ing. Eléctrica y Mecatrónica, Ing. Industrial, Ing. Química y Bioquímica, Ing. Sistemas y Computación, División de Estudios de Posgrado e Investigación y Desarrollo Académico, este último departamento es el coordinador general del proceso de la Evaluación del Desempeño Docente. Respecto a la estrategia para la recolección de los datos, se buscó el apoyo institucional de las áreas académicas para la distribución del formulario y la asistencia del equipo de investigadores a reuniones de academia para solicitar el apoyo de los docentes y poder concluir con éxito la recolección de datos.

Esta investigación se desarrolló bajo el enfoque cualitativo y cuantitativo, no experimental y de alcance exploratorio y descriptivo. Se contempla de carácter exploratorio y descriptivo respecto al análisis que se hace de los mecanismos establecidos en el Sistema para la evaluación del desempeño docente del Instituto Tecnológico de Tepic desde la perspectiva de los docentes y de los administradores del proceso de la CEDD, analizando específicamente el Instrumento de evaluación al desempeño docente, así como el nivel de aceptación, para analizar el impacto de la evaluación, sus fortalezas y áreas de mejora.

Se acudió a la investigación documental en textos y bases de datos especializadas para describir las competencias del perfil docente más importantes que expertos en la materia han documentado; así como también para fundamentar el marco teórico en el tema de la evaluación del desempeño, toda esta información forma parte del marco referencial de esta investigación. Se recurrió a la técnica de investigación de campo para recoger la opinión de los docentes y administradores del proceso de la CEDD respecto a su opinión conocimiento y aceptación que tienen acerca del Instrumento de Evaluación. Su enfoque es cualicuantitativo con la finalidad de identificar el nivel de aceptación, la investigación por su ubicación temporal es transversal por que se realizará solo única ocasión, de acuerdo al conocimiento del problema.

1SSN: $2007-8870$

Universidad de Sonora los contenidos de este articulo ertin bajo una licendia de Creative Commons Atribueión No Comercial - Sin Obera Derivada 4 , Ointernaciona 
Para el diseño del instrumento de recolección de datos que en lo sucesivo se denominará Anexo 1, se analizó el CEDD y se diseñó un cuestionario con preguntas cerradas y una abierta, tomando como base las variables: opinión sobre el instrumento de evaluación al desempeño docente y su aplicación, y la participación del docente. En relación a este instrumento, se realizó la prueba piloto del instrumento de recolección a 48 docentes del área de Ciencias EconómicoAdministrativas del Instituto Tecnológico de Tepic para analizar su confiabilidad, los datos fueron procesados en el software SPSS, dando como resultado un Alfa de Cronbach de .933, que se interpreta como un nivel muy alto. En cuanto a los nueve administradores del proceso se aplicó un cuestionario con preguntas abiertas y cerradas (Anexo 2), en donde se obtuvo información respecto al uso de los resultados obtenidos en la evaluación del desempeño docente.

\section{Recolección de Datos.}

Los cuestionarios se codificaron en un formulario de google para los docentes, una vez capturada la información a través de un enlace con acceso directo al formulario donde se obtuvieron los gráficos de cada una de las preguntas y se realizó el traslado de los datos a Excel, de ahí se procede a la interpretación de cada uno de los resultados por pregunta. De la misma manera se realizó un formulario para recabar la información de los administradores del proceso de la CEDD, que fueron preguntas cerradas y abiertas. En las preguntas abierta se realizaron tablas que sirvieron de apoyo para analizar e identificar la popularidad de las respuestas en el caso de las preguntas donde se solicitó elegir un orden de importancia de las respuestas propuestas. Para el análisis de la información se utilizarán técnicas de resumen, síntesis y la comparación, y para la presentación de resultados el uso de gráficos y tablas. Para el procesamiento de las preguntas abiertas, se utilizó el software NVIVO de investigación cualitativa para identificar las respuestas más frecuentes; también se agruparon las respuestas en categorías para resumir las propuestas de los estudiantes en relación al instrumento y proceso de evaluación de los docentes.

Al coordinador general del proceso de evaluación del desempeño docente, se le aplicó de forma virtual sincrónica una entrevista para conocer los aspectos generales del proceso de evaluación, como tiempo de duración, periodo de aplicación, difusión de los resultados, validez del 
instrumento, retroalimentación de los resultados obtenidos, seguimiento y áreas de mejora tanto del instrumento como del proceso en sí mismo.

\section{Hallazgos}

El resultado del cuestionario aplicado a los docentes del Instituto Tecnológico de Tepic se realizó de manera proporcional a los diferentes departamentos académicos. La plantilla de personal docentes es 299 el tamaño de la población y el tamaño de la muestra es de 169 docentes encuestados, se superó la información recabada fue de 183 docentes y con ello se logró obtener más información de la esperada, en la Tabla 2, se muestra la cantidad y porcentaje de docentes por departamento académico que contestaron la encuesta.

Para efecto de este documento sólo se contemplan el Apartado 1 y el Apartado 2 del cuestionario realizado y aplicado a los docentes para efecto de este proyecto. El Apartado 1, se relaciona al diseño del cuestionario, que incluye la cantidad de preguntas del instrumento, escala de valoración utilizada, claridad y comprensión textual de las preguntas, competencias que evalúa, modalidad de la evaluación (en línea) y periodo de aplicación, de forma general y para una mejor apreciación de la información se presentan la Tabla 4 y la Figura 1; el Apartado 2, permite valorar los beneficios, pertinencia y validez de los resultados del instrumento del CEDD.

Apartado 1 En relación al diseño del cuestionario, evalúe los aspectos que se detallan a continuación.

* Para el criterio "Cantidad de preguntas del cuestionario", el 26\% de los docentes, manifestaron estar satisfechos, mientras que el $8 \%$ consideran la cantidad como eficiente.

* Para el criterio "Escala de valoración utilizada", el 31\% de los docentes, manifestaron estar satisfechos, mientras que el $4 \%$ consideran la escala como algo eficiente.

* Para el criterio "Claridad y comprensión textual de las preguntas", el 27\% de los docentes, manifestaron estar satisfechos, mientras que el 5\% lo considera deficiente. 
* Para el criterio "Competencias que evalúa el CEDD", el 25\% de los docentes, manifestaron encontrarse satisfechos con las competencias que evalúa, mientras el 3\% las considera deficientes.

* Para el criterio "Periodo en que se aplica la evaluación", el 39\% de los docentes, manifestaron estar satisfechos con la modalidad de la evaluación, mientras que sólo el $1 \%$ considera deficiente la modalidad.

* Para el criterio "Periodo en que se aplica la evaluación", el 22\% de los docentes, manifestaron estar satisfechos con el periodo en que se aplica, mientras que el $6 \%$ está en desacuerdo en el periodo en que se aplica

\section{Tabla 4}

Resultados Apartado 1

\begin{tabular}{|c|c|c|c|c|c|c|}
\hline Criterios & Muy bien & Bien & Regular & Deficiente & Muy bien & Deficiente \\
\hline Cantidad de preguntas en el cuestionario & 48 & 72 & 48 & 15 & $26 \%$ & $8 \%$ \\
\hline Escala de valoración utilizada & 57 & 84 & 34 & 8 & $31 \%$ & $4 \%$ \\
\hline $\begin{array}{l}\text { Claridad y comprensión textual de las } \\
\text { preguntas }\end{array}$ & 50 & 75 & 49 & 9 & $27 \%$ & $5 \%$ \\
\hline Competencias que evalúa el CEDD & 46 & 88 & 43 & 6 & $25 \%$ & $3 \%$ \\
\hline $\begin{array}{l}\text { Modalidad en que se aplica la } \\
\text { evaluación }\end{array}$ & 72 & 78 & 31 & 2 & $29 \%$ & $1 \%$ \\
\hline Periodo en que se aplica la evaluación & 40 & 63 & 27 & 11 & $22 \%$ & $6 \%$ \\
\hline
\end{tabular}




\section{Figura 1}

Resultados Apartado 1

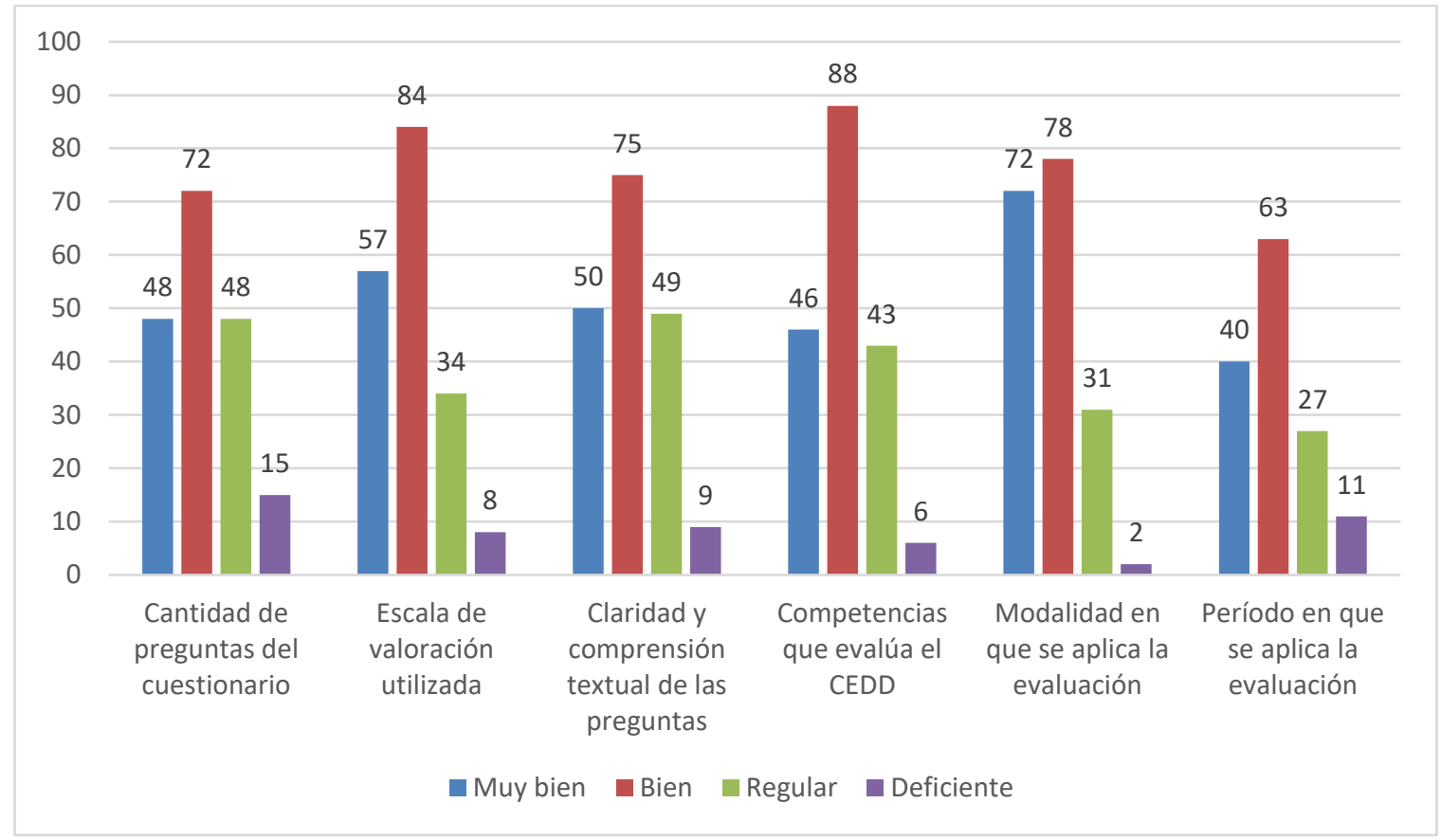

Apartado 2. En cada una de las siguientes afirmaciones, indique el grado en que usted está de acuerdo con ellas, los resultados obtenidos se muestran de forma integral en la Tabla 5 y en la Figura 2.

- Para el criterio "el CEDD es un mecanismo que ayuda a identificar las fortalezas y áreas de oportunidad del desempeño docente", 58 docentes dijeron estar totalmente de acuerdo, y 11 en desacuerdo.

* Para el criterio "las preguntas del cuestionario son las adecuadas para evaluar las competencias del docente", 43 docentes indicaron estar totalmente de acuerdo, mientras que 9 de ellos, estuvieron en desacuerdo.

* Para el criterio "el resultado del CEDD es importante", 73 docentes estuvieron totalmente de acuerdo, y 10 totalmente en desacuerdo. 
* Para el criterio "las competencias que evalúa el CEDD son necesarias e importantes", 67 docentes asumieron estar totalmente de acuerdo, mientras que 6 opinaron lo contrario.

Para el criterio "los resultados del instrumento son válidos", 43 docentes estuvieron totalmente de acuerdo, y 16 de ellos coincidieron en totalmente en desacuerdo.

Para el criterio "el CEDD es un instrumento claro para los estudiantes", 44 docentes manifestaron estar totalmente de acuerdo mientras que 19 de ellos, dijeron estar totalmente en desacuerdo.

\section{Tabla 5}

\section{Resultados Apartado 2}

\begin{tabular}{lcccc}
\hline Criterios & $\begin{array}{c}\text { Totalmente } \\
\text { de acuerdo }\end{array}$ & $\begin{array}{c}\text { De } \\
\text { acuerdo }\end{array}$ & $\begin{array}{c}\text { En } \\
\text { desacuerdo }\end{array}$ & $\begin{array}{c}\text { Totalmente en } \\
\text { desacuerdo }\end{array}$ \\
\hline $\begin{array}{l}\text { El CEDD es un mecanismo que ayuda a identificar las } \\
\text { fortalezas y áreas de oportunidad del desempeño de los }\end{array}$ & 58 & 91 & 23 & 11 \\
docentes. & & & \\
Las preguntas del cuestionario son las adecuadas & 43 & 85 & 46 & 9 \\
El resultado del CEDD es importante & 73 & 83 & 17 & 10 \\
Las competencias que evalúa el CEDD son necesarios e & 67 & 82 & 28 & 6 \\
importantes & & 43 & 82 & 42 \\
Los resultados del instrumento son válidos & 44 & 74 & 46 & 16 \\
El CEDD es un instrumento claro para los estudiantes & & & 19 \\
\hline
\end{tabular}




\section{Figura 2}

Resultados Apartado 2

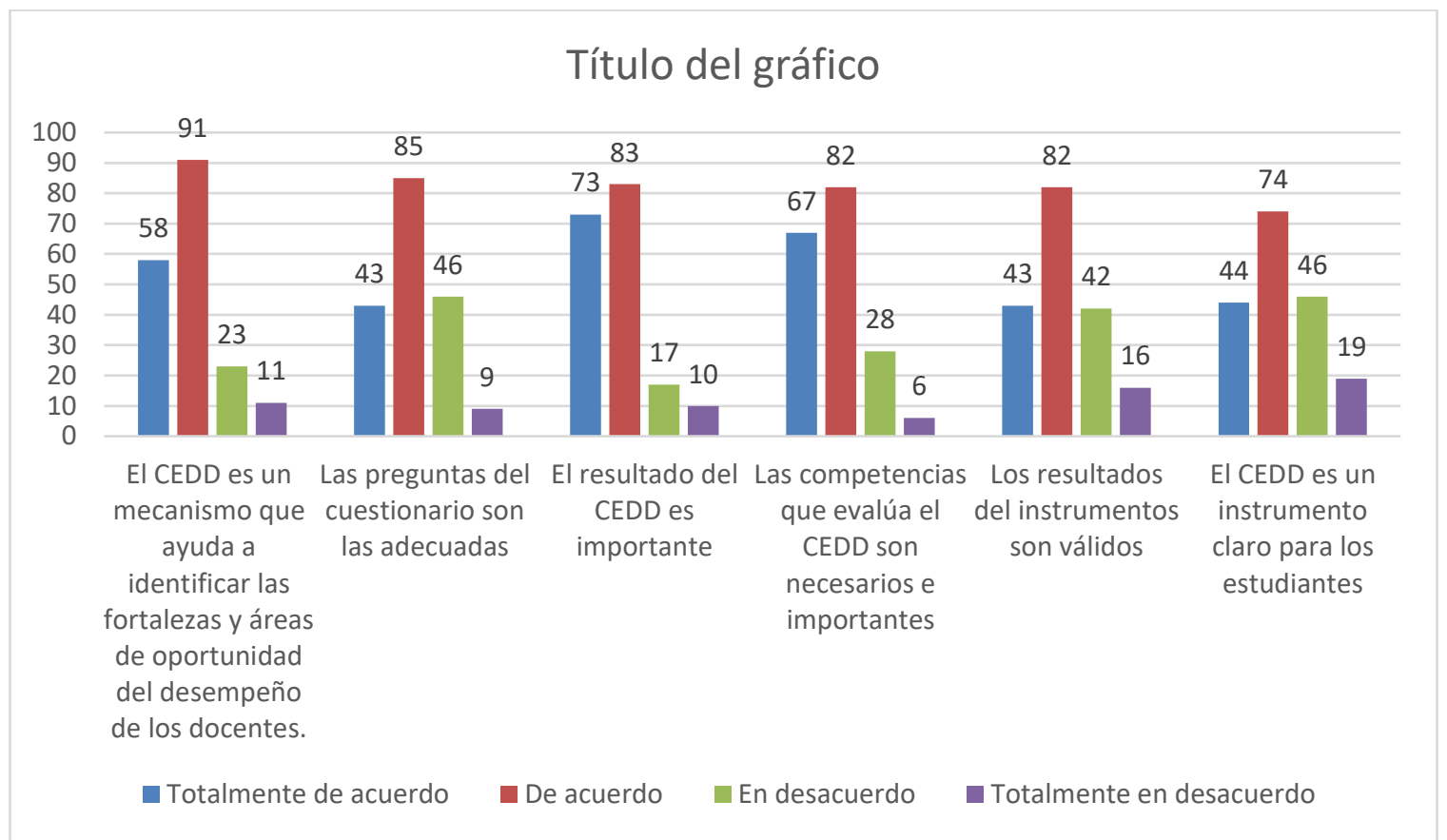

\section{Conclusiones}

En relación al cumplimiento de los objetivos planteados en la investigación sobre la aceptación del instrumento por parte de los docentes del Instituto Tecnológico de Tepic, en relación al Apartado 1, se puede decir que las preguntas del CEDD cuentan con un alto rango de aceptación, ya que la mayoría de la valoración que se le dio fluctúa entre "bien y muy bien" de acuerdo a la escala utilizada. Sin embargo, al analizar los resultados se puede concluir que la cantidad de preguntas, la claridad y comprensión de las mismas, así como las competencias que evalúan, deben ser revisadas para mejorarlas; asimismo, aunque en menor ponderación, la escala de valoración, la modalidad de aplicación y el periodo de aplicación también deben ser revisados. 
Respecto al Apartado 2, se destaca una gran aceptación del instrumento; sin embargo, se destaca que la pertinencia de las preguntas, la validez de los resultados y la claridad del instrumento para los estudiantes son elementos que deben ser analizados a profundidad.

A partir de los resultados se pueden plantear las siguientes recomendaciones: aplicar la encuesta en el tiempo adecuado para poder generar acciones correctivas y no al final del curso; actualizar el cuestionario para asegurar la pertinencia de las preguntas acorde al contexto del momento de su aplicación; estandarizar como parte del proceso acciones remediales; concientizar a los estudiantes de la importancia de sus respuestas; y reducir el número de preguntas.

En consecuencia, podemos decir que estructurar un proceso de evaluación del desempeño en la organización es un proceso continuo de aprendizaje, en el que hay que ir incorporando nuevos elementos que condicionan o impulsan el desempeño del personal y en el que hay que ir descartando aquellos elementos que la experiencia nos va demostrando que tienen poca utilidad para promover el propósito que se persigue con el proceso de evaluación del desempeño: enfocar al personal en los resultados que la organización espera de sus puestos. Por consiguiente, toda evaluación debiera ser un proceso para estimular o juzgar el valor, la excelencia o las cualidades de una persona. La evaluación de los individuos que desempeñan papeles dentro de una organización se hace aplicando varios procedimientos que se conocen por distintos nombres, como evaluación del desempeño, calificación de méritos, evaluación de los empleados, informes de avance, evaluación de la eficiencia en las funciones, etc.

La evaluación del desempeño es un proceso gerencial clave para cualquier organización, y al mismo tiempo una herramienta de la gestión de recursos humanos, ya que suministra información valiosa para enfocar acciones pertinentes que impacten en la mejora del desempeño de los colaboradores, y por consecuencia en los resultados organizacionales, contribuye a que, tanto los empleados, como sus jefes o supervisores orienten sus conocimientos, su tiempo, sus esfuerzos, sus recursos y acciones hacia aquellos resultados clave que determinan los resultados que alcanza una organización cuando pretende lograr sus objetivos. 
La evaluación del desempeño no es un objetivo, sino un medio para mejorar los resultados de los recursos humanos de la organización, y por consecuencia los resultados organizacionales. Cuando se evalúa el desempeño de los recursos humanos se efectúa una revisión acerca de la eficacia con que este se desempeña en su puesto; es decir una evaluación sobre que tanto los recursos humanos se acercan a los desempeños esperados y necesarios para que la organización alcance sus metas. Y, esta medición al mismo tiempo representa una excelente oportunidad para también investigar porque los empleados no alcanzan el desempeño esperado, o en su caso superan eficazmente esos resultados que la organización espera en sus puestos de trabajo. De esta investigación, se desprende información valiosa respeto al instrumento utilizado en los institutos tecnológicos, mismo que debe ser actualizado y valorado constantemente para mejorar su eficiencia y eficacia.

\section{Referencias}

Gobierno de la Republica. (2019). Anexo XVIII-1 Anexos al Plan Nacional de Desarrollo 20192024. Gaceta Parlamentaria, XXII(5266). Retrieved from http://gaceta.diputados.gob.mx/PDF/64/2019/abr/20190430-XVIII-1.pdf

Gómez López, L. F., \& Valdés, M. G. (2019). La evaluación del desempeño docente en la educación superior. Propósitos y Representaciones, 7(2). https://doi.org/10.20511/pyr2019.v7n2.255

PTA Tecnm.(n.d.).Programa_de_Trabajo_Anual_2019_del_TecNM.pdf.

Tejedor, F. J. (2012). Valuación del. 5.

Arámburo Vizcarra, Vicente, \& Luna Serrano, Edna. (2013). La influencia de las características, profesor y del curso en los puntajes de evaluación docente. Revista mexicana de investigación educativa, 18(58), 949-968. Recuperado el 28 de octubre de 2017, de http://www.scielo.org.mx/ scielo.php?script=sci_arttext\&pid=S140566662013000300012\&lng=es\&tlng=es.

Certo, S. (1994). Administración Moderna. México. Editorial Pearson.

Chiavenato, I. (2000). Administración de Recursos Humanos. Colombia. Mc Graw-Hill Interamericana, S.A. 
Chiavenato, I. (2009). Gestión del talento humano. México. Editorial McGraw-Hill.

Cortés, E., \& Campos, M., \& Moreno, M. (2014). Priorización de las dimensiones de evaluación al desempeño docente por el estudiante, en tres áreas del conocimiento. Formación Universitaria, 7 (2), 3-10.

Dirección General de Educación Superior Tecnológica. (2006). Manual de los procesos de evaluación del desempeño docente y del ingreso de aspirantes a la Educación Superior Tecnológica. Recuperado de: http://www.itpachuca.edu.mx

(2012). Modelo Educativo para el Siglo XXI. Formación y Desarrollo de Competencias profesionales. Recuperado de http://tecnm.mx/modeloeducativo.pdf

-------- (2013). Evaluación Docente con enfoque en Competencias. Recuperado de: http://tecnm.mx/docencia/evaluaciondocente

Fernández, D. J. y otros (2006), Modelos de Calidad, en la dirección escolar ante los retos del siglo XXI, Ed. Síntesis.

García, María. (2001) “La importancia de la evaluación del desempeño”. Revista proyecciones. Año 2 Número 9.

García, T., Espinoza, S.y Sandoval, B. (2017). Capacitación Docente del Instituto Tecnológico de Tepic y la Relación con la Evaluación y Desempeño. Coloquio de investigación multidisciplinaria Journal CIM. Volumen 5, número 2.

Gobierno de la República. (2013). Plan Nacional de Desarrollo 2013-2018. México, D. F. Recuperado de: http://tecnm.mx/informacion/documentos-del-snest

Juárez, O. (2014). Administración de la compensación, sueldos, salarios, incentivos y prestaciones. México. Grupo Editorial Patria, S. A. de C. V.

Martínez, Chairez, G., \& Guevara, Araiza, A., \& Valles, Ornelas, M. (2016). El desempeño docente y la calidad educativa. Ra Ximhai, 12 (6), 123-134.

Robalino, M. (2005). ¿Actor o protagonista? Dilemas y responsabilidades sociales de la profesión docente. En OREALC/UNESCO Editor. Protagonismo docente. En el cambio educativo. Revista Prelac N¹. (6-23). Santiago: AMF

Romo Martínez, J. (2013). Evaluación Comprehensiva del Desempeño Docente. Conciencia Tecnológica, (46), 52-55. 
Rueda, M., Elizalde, L. y Torquemada, A. (2003, julio-septiembre). La evaluación de la Docencia en las universidades mexicanas. Revista de la Educación Superior 127, 7177.

Tecnológico Nacional de México. (2015). Instrumento de la Evaluación Departamental. Recuperado de: http://tecnm.mx/docencia/evaluaciondocente.

-(2014) Programa Institucional de Innovación y Desarrollo 2013-2018. México. Werther, William B. (2000). Administración de Personal y Recursos Humanos. México. Editorial McGraw-Hill

Espinoza Morales, S. (2021). La Evaluación del Desempeño Docente desde la perspectiva del docente y de los administradores del proceso : La evaluación es el principal elemento para mejorar el quehacer docente. Revista De Investigación Académica Sin Frontera: División De Ciencias Económicas Y Sociales, (35). https://doi.org/10.46589/rdiasf.vi35.402
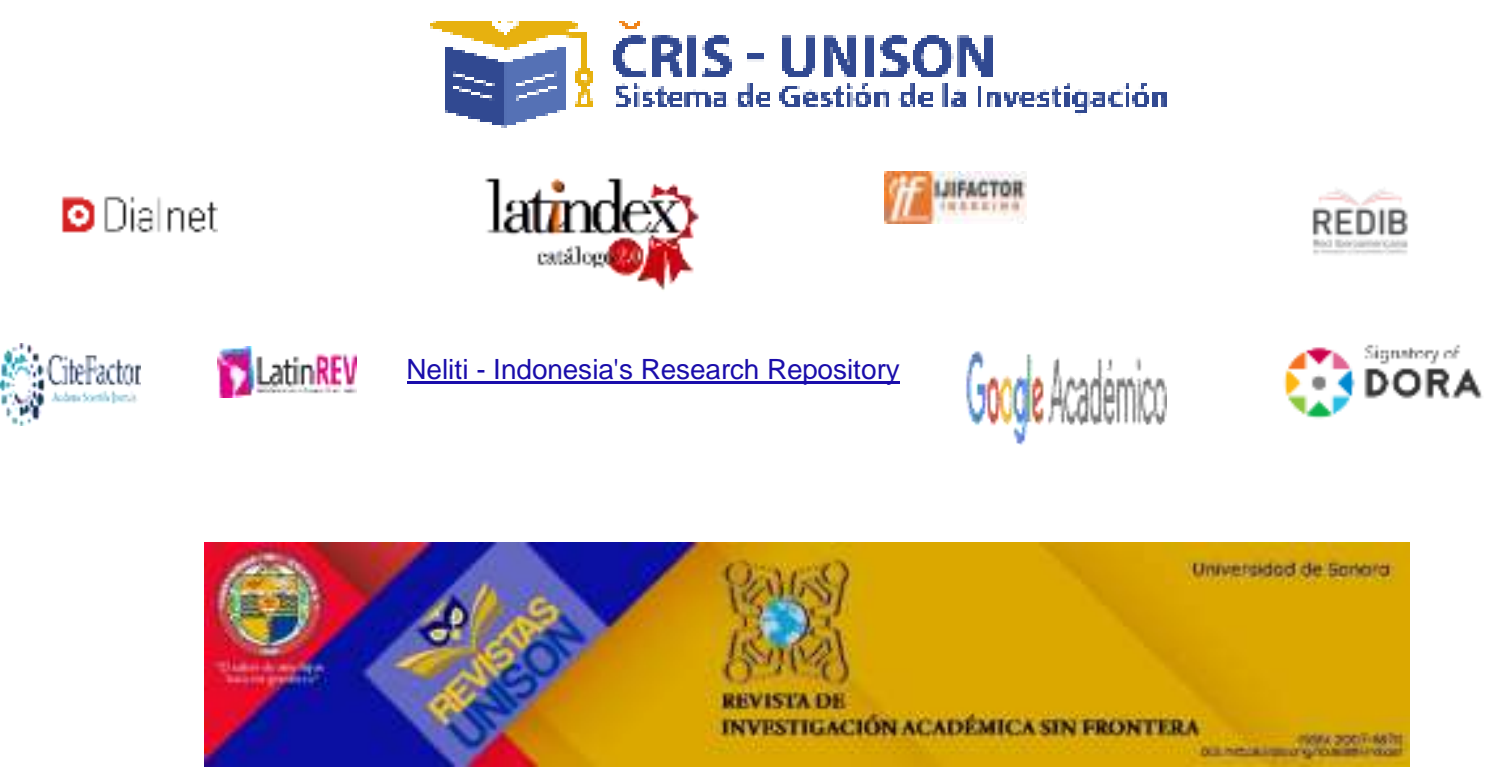